Hendricks, R. W., de Lorenzo, J. D., Glass, F. M. \& ZEDLER, R. E. (1973). J. Appl. Cryst. 6, 129-132.

Hendricks, R. W. \& Schmidt, P. W. (1967). Acta Phys. Austr. 26, 97-122.

Hendricks, R. W. \& Schmidt, P. W. (1973). Acta Phys. Austr. 37, 20-30.

Hendricks, R. W. \& Shaffer, L. B. (1971). Some Properties of Octafluorocylobutane $\left(\mathrm{C}_{4} \mathrm{~F}_{8}\right)$ of Interest in $X$-Ray Absolute Intensity Experiments, USAEC Report ORNLTM-3407, Oak Ridge National Laboratory, Oak Ridge, Tennessee.

Kratky, O., Pilz, I. \& Schmitz, P. J. (1966). J. Colloid Interface Sci. 21, 24-34.
Malet, G., Cabo;, C., Escande, A. \& Delord, P. (1973). J. Appl. Cryst. 6, 139-144.

Pilz, I. \& KRATKY, O. (1967). J. Colloid Interface Sci. 24, 211-218.

Pilz, I. (1969). J. Colloid Interface Sci. 30, 140-144.

Shaffer, L. B. \& Beeman, W. W. (1970). J. Appl. Cryst. 3, 379-384.

Smith, P. J. (1971). FITLOS: A Fortran Program for Fitting Low-Order Polynomial Splines by the Method of Least Squares, National Aeronautics and Space Administration, Report NASA-TN-D6401, Lewis Research Center, Cleveland, Ohio.

WeinBerg, D. L. (1963). Rev. Sci. Instrum. 34, 691-696.

\title{
Small-Angle X-ray Scattering and Biological Structures
}

\author{
BY W. W. BEEMAN \\ Biophysics Laboratory, University of Wisconsin, Madison, Wisconsin 53706, U.S.A.
}

The three years since the Second International Small-Angle X-ray Scattering Conference have seen a great many important applications of small-angle X-ray scattering to the determination of biological structures. There are many more than can be reviewed in a single lecture. Fortunately, the recent comprehensive review by Kratky \& Pilz [Quart. Rev. Biophys. (1972). 5, 481], makes it appropriate that some selectivity be exercised in this presentation. Research on biological membranes and on isotropic solutions of nucleic acids rather than studies of the size and shape of globular molecules is emphasized. Small-angle X-ray investigations of membranes and nucleic acids share certain similarities of approach which the authors believe will become increasingly important for a variety of biological systems. For instance the uniform-electron-density approximation is usually not of interest. One seeks information on the variation of electron density within and near the particle. Commonly there is a great deal of structural information available from chemical or other evidence and the role of small-angle X-ray scattering is to choose among a small number of possible structures or to investigate deviations from established structures produced by a variety of chemical and physical environments. The comparison of experimental scattering curves with those computed from assumed structures may be of great assistance. If one attempts a detailed comparison of experimental and computed scattering curves and if, as is frequently the case, fairly small equivalent Bragg spacings are of interest the proper subtraction of the solvent background scattering becomes a difficult problem. The solvent cannot be treated as a homogeneous medium of uniform electron density. Some aspects of this problem will be discussed briefly. 